\title{
Weibull Model Development for Fatigue Crack Growth
}

\author{
Bobby Day and T. Goswami \\ Mechanical Engineering Department \\ Arkansas Tech University \\ Russellville, AR 72801, USA
}

\section{ABSTRACT}

This effort is an attempt to "Weibullize" fatigue crack growth rate data as a function of stress intensity range $(\Delta \mathrm{K})$. It is not a classical Weibull application in that it does not address a population but rather treats rate of crack growth as a fraction of the highest rate of crack growth rate in the data set (usually the final data point of the set) as the "failed fraction of a population." All $\mathrm{da} / \mathrm{dN}$ values are converted to a percentage or fraction of the $100 \%$ data value, processed into a linear relationship and plotted against the natural log of the corresponding $\Delta \mathrm{K}$ value.

The crack growth rate data ( $\mathrm{da} / \mathrm{dN}$ vs. $\Delta \mathrm{K})$, when converted to a Weibull type plot, can be fitted with a linear curve with $R^{2}$ values in the 0.99 range and characteristic Weibull parameters $\beta$ and $\eta$ can be successfully determined. For the data analyzed, the mean value determined for $\beta$ is 2.86 with a standard deviation of 0.36 . However, data from sets of apparently similar specimens produced more closely packed results. The data sets that could not be fit with the Weibull distribution (4 of 32 sets) appeared to have been corrupted, possibly by sorting the data in the spread sheet without including all of the columns. $R^{2}$ values of $>$ 0.99 were obtained for the linear curve fit of 15 of 28 data sets. $R^{2}$ values $>0.95$ were obtained for an additional 7 while the remaining 6 were $>0.64$

\section{INTRODUCTION}

Weibull analysis methodology has been reviewed for other applications and extended to analyze fatigue crack growth.

The Weibull distribution is defined mathematically as

$$
F(t)=1-e^{-((t-\gamma / n) / \beta}
$$


The classical use of the Weibull distribution is to provide failure projections for a population based on observed failures in that population. In this application:

$\mathrm{F}(\mathrm{t}) \quad=$ failed fraction of a population

$t=$ time of failure

$\gamma \quad=$ starting point or origin of the distribution if other than $t_{0}$

$\eta \quad=$ characteristic life (the time or number of cycles at which $63.2 \%$ of the population is expected to have failed (Analogous to RC time constant in electronics). $63.2 \%$ is given by $F(t)=1-e^{-1}$ when $t$ $=\eta$

$\beta \quad=$ slope or shape parameter

$\beta$ and $\eta$ are the characteristic parameters of a 2 parameter Weibull application. Adding $\gamma$ makes a 3 parameter application.

By letting $\gamma=$ zero and manipulating the above expression into the form of the straight line equation

$\mathrm{Y}=\mathrm{MX}+\mathrm{B}$

such that $\underline{\ln \ln \{1 /(1-F(t))\}}=\beta \underline{\ln t}-\beta \ln \eta$

and regarding $\underline{\ln \ln \{1 /(1-F(t))\}}$ as $Y$ and $\underline{\ln } \underline{t}$ as $X$

(see Appendix 1) we can now plot failure data $(\mathrm{da} / \mathrm{dN}=$ crack growth rate) vs. time (cycles, $\Delta \mathrm{K}$, etc.). Data that approximates a straight line when processed in this fashion can be analyzed to determine the associated values of the Weibull characteristic parameters. If the data plot does not approximate a straight line, it may indicate the presence of multiple failure modes and/or an offset in the $\mathrm{X}$ axis initiation of the distribution $(\gamma$ not $=0$ ).

By using the Excel "Insert Trend Line" function, the data can be curve fit and the equation of the resulting line displayed. $\beta$, the shape factor, is the coefficient of $X$ (slope of the line) from this equation. The final term in the straight line equation (the $Y$ axis intercept) would appear to be useful in determining $\eta$, the characteristic life, but due to a $Y$ scale shift to present the data in a "Weibull graph paper" format, a different method is used. The $Y$ value representing $0.632 F(t)$ is placed in the obtained equation as a known $Y$ value and the equation is solved for the corresponding $\mathrm{X}$ value. This yields the natural log of the Eta value of $\Delta \mathrm{K}$.

If the data fails to plot as a straight line due to an offset in the start of the distribution, shifting the "time" scale or $\mathrm{X}$ axis values until the curve straightens out allows a quantification of the offset. The spreadsheet provides for use and definition of the value of the third Weibull parameter, distribution initiation offset or $\gamma$. The $\Delta \mathrm{K}$ offset for the origin of the distribution was varied and the variation of the curve fit $\mathrm{R}^{2}$ value was observed. The offset value at which the $\mathrm{R}^{2}$ value peaked is suggested as an approximation of the $\gamma$ value. However, human observation of the variation in the shape of the lower end of the curve seemed to provide better results than the $\bar{R}^{2}$ value peak as $R^{2}$ evaluates the quality of the fit over the entire length of the curve and not just the lower end. 
The $\beta$ or "shape factor" values obtained were fairly consistent while there was considerable spread in the $\eta$ or "characteristic life" parameter. This is believed to be due to the variety of specimens in the data (various initial crack lengths and/or specimen geometry). The Weibull parameter values obtained can then be used in various Weibull equations to provide predictions concerning the expected behavior of the population.

The rate of crack growth as a function of $\Delta \mathrm{K}$ is known to occur in three regions (Ref. 2) as shown in the figure below.

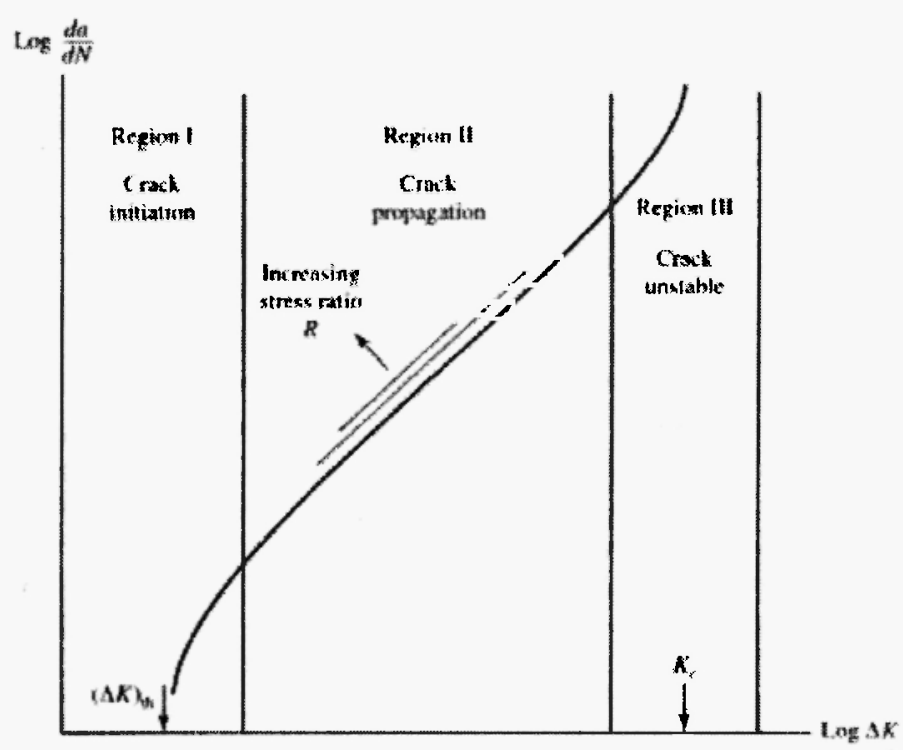

Fig. 1: Fatigue Crack Growth Regions (Ref. 2)

The data evaluated covered primarily region II with Region I or portions of it included in some cases. Some data sets also seemed to contain the onset of Region III. In general, the data plotted was limited to Region II as the area of interest. In some cases, Regions I and II were plotted and offset varied to quantify threshold $\Delta \mathrm{K}$.

The methodology and resulting plots are described and provided in the following pages.

\section{CRACK GROWTH RATE VS. $\triangle$ K PROCESS DEVELOPMENT}

The Microsoft Excel spreadsheet program was used to process all data in this effort. da/dN data as a function of $\Delta \mathrm{K}$ for 32 test specimens was provided by others. For development of this process, the data set for the specimen designated CT11 was used. After development of the process using CT11, data from the remaining specimens was analyzed. Table I provides a summary of the analysis results. Table 2 is the CT11 spread sheet and was used interactively with the resulting graph (Figure 2) to evaluate the quality of the resulting plot and to define the values of $\beta$ and $\eta$. It was also used to explore the effect of variations of the value of $\gamma$. For specimen CT11, $\gamma$ was set to the value (4) that produced the straightest line in the plot of data as indicated by the curve fit $R^{2}$ value. 
Table I

\begin{tabular}{|c|c|c|c|c|c|}
\hline Specimen & $\overline{\text { Beta }}$ & Eta & Gamma & $\mathbf{R}^{2}$ & Remarks \\
\hline CT11 & 2.7983 & 42.38 & 0 & 0.9947 & \\
\hline CT11 & 2.3095 & 39.37 & 4 & 0.9957 & \\
\hline CT12 & 2.7613 & 42.91 & 0 & 0.9968 & \\
\hline CT12 & 2.5286 & 41.73 & 1.5 & 0.9969 & \\
\hline CT13 & 3.0065 & 37.3 & 0 & 0.9937 & Peak $R^{2}$ at 0 and -1 \\
\hline CT14 & 2.7918 & 45.46 & 0 & 0.991 & \\
\hline CT15 & 2.7896 & 42.21 & 0 & 0.9937 & \\
\hline CT16 & 3.42 & 30.68 & 0 & 0.9717 & \\
\hline CT18 & 3.1392 & 29.41 & 0 & 0.9913 & Bottom 15 and Top 3 Points Censored \\
\hline CT22 & & & & & Corrupted Data \\
\hline CT23 & 3.8298 & 27.82 & 0 & 0.9824 & Does not linearize well \\
\hline CT24 & & & & & Corrupted Data \\
\hline CT24+1 & 2.1814 & 55.9 & 0 & 0.9529 & \\
\hline CT2 & 2.7153 & 54.6 & 0 & 0.9984 & \\
\hline CT4 & 2.7506 & 40.31 & 0 & 0.9463 & \\
\hline CT36 & 2.6823 & 37.7 & 0 & 0.6497 & \\
\hline CT37 & 3.0598 & 42.12 & 0 & 0.84 & \\
\hline CT31 & 2.894 & 29.39 & 0 & 0.7935 & \\
\hline CT32 & & & & & Corrupted Data \\
\hline 33 & & & & & Corrupted Data \\
\hline $\mathrm{CC} 12$ & 3.0974 & 30.21 & 0 & 0.9893 & \\
\hline $\mathrm{CC} 21$ & 2.6827 & 66.91 & 0 & 0.9978 & \\
\hline $\mathrm{CC} 26$ & 3.1276 & 33.75 & 0 & 0.9904 & \\
\hline $\mathrm{CCl6}$ & 2.9385 & 56.69 & 0 & 0.9903 & \\
\hline $\mathrm{CC} 16$ & 2.3538 & 53.25 & 5 & 0.9924 & \\
\hline $\mathrm{CC17}$ & 2.684 & 67.91 & 0 & 0.9937 & Lowest 7 data points censored \\
\hline $\mathrm{CC17}$ & 2.9637 & 64.6 & 0 & 0.9604 & No data censored \\
\hline $\mathrm{CCl}$ & 1.6587 & 68.25 & 9.45 & 0.9858 & No Data Censored \\
\hline $\mathrm{CC} 18$ & 2.6813 & 71.23 & 0 & 0.9931 & @,6 \\
\hline $\mathrm{CC} 4$ & 2.2727 & 91.99 & 0 & 0.9917 & Upper 4 points censored \\
\hline $\mathrm{CC} 4$ & 2.4114 & 87.3 & 0 & 0.9817 & Uncensored \\
\hline $\mathrm{CC5}$ & 2.3883 & 89.57 & 0 & 0.991 & Region 2 \\
\hline $\mathrm{CC} 6$ & 2.3848 & 93.17 & 0 & 0.9822 & Region 2 \\
\hline $\mathrm{CC} 23$ & 2.7339 & 108.8 & 0 & 0.9946 & top 4 points censored \\
\hline $\mathrm{CC} 23$ & 2.8978 & 99.33 & 0 & 0.9908 & uncensored \\
\hline $\mathrm{CC} 24$ & 2.7352 & 69.37 & 0 & 0.9792 & uncensored \\
\hline $\mathrm{CC} 24$ & 2.5643 & 74.24 & 0 & 0.989 & Top 4 points censored \\
\hline $\mathrm{CC} 25$ & 3.1174 & 37.75 & 0 & 0.9806 & uncensored \\
\hline $\mathrm{CC} 28$ & 3.3399 & 53.23 & 0 & 0.6547 & Suspect data; highly scattered \\
\hline $\mathrm{CC} 29$ & 3.527 & 35.5 & 0 & 0.9259 & Suspect data; scattered \\
\hline $\mathrm{CC} 30$ & 2.8767 & 38.18 & 0 & 0.8198 & Suspect data; scattered \\
\hline
\end{tabular}


Table II
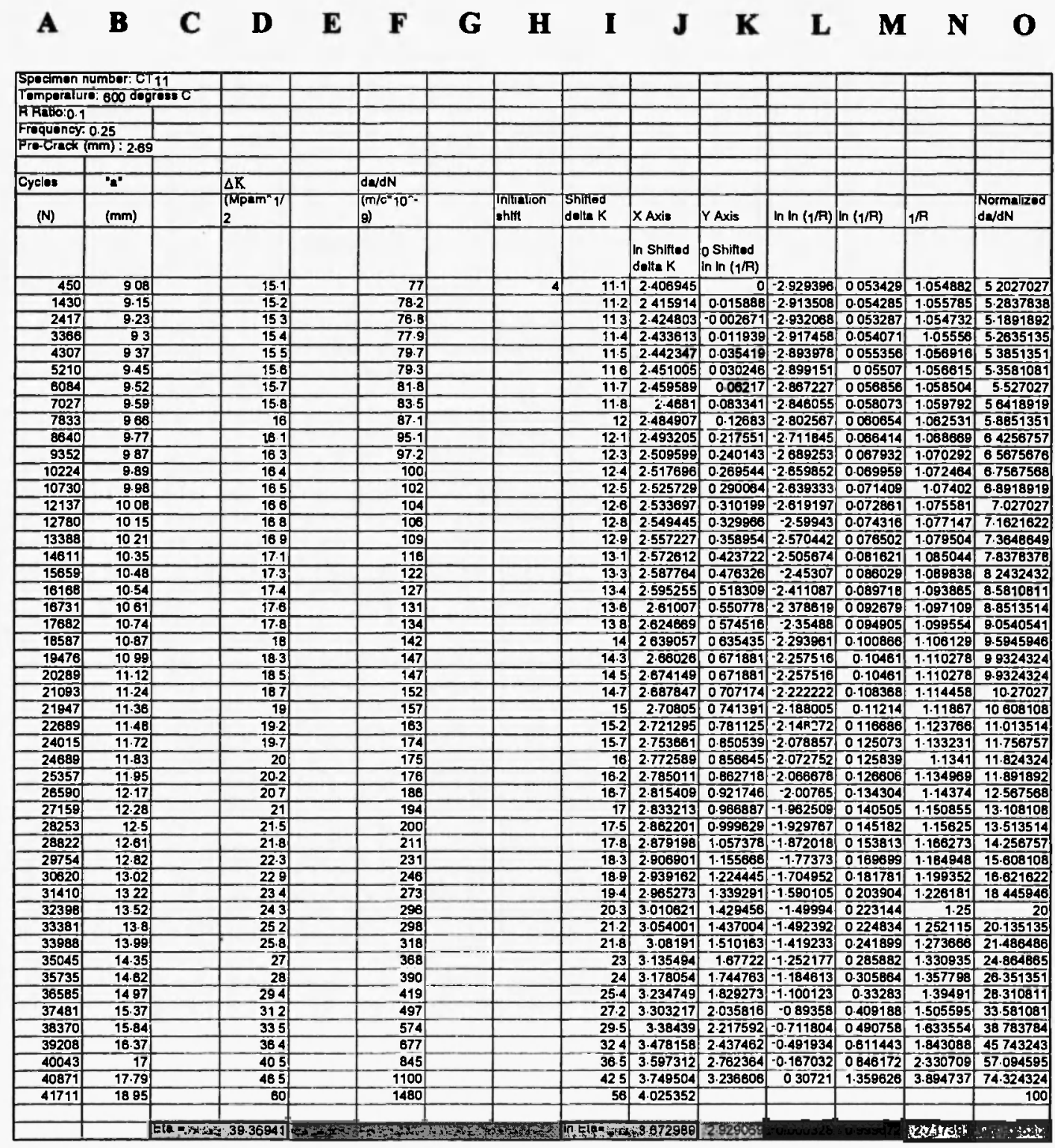

The source data for specimen CTII appears in columns A through F of Table II. The data processed is $\Delta \mathrm{K}$ in column $\mathrm{D}$ and $\mathrm{da} / \mathrm{dN}$ in column $\mathrm{F}$.

The initial part of the process is to develop the Weibull distribution values representing $\Delta \mathrm{K}$ to be plotted on the $\mathrm{X}$ axis. To do this, a distribution initiation adjustment $(\gamma)$ is provided in column $\mathrm{H}$. Column I, labeled "Shifted delta $K$ " is then the value from the same row in column D minus the value of $\gamma$ entered in column $H$. Column $\mathrm{J}$ contains the natural logs of the values from the same row in column I and which are the values plotted on the $\mathrm{X}$ axis. The scale values for $\ln \Delta \mathrm{K}$ were suppressed by changing them to background color. $\mathrm{A}$ $\Delta \mathrm{K}$ scale was created by plotting the natural $\log$ of values of interest as data with corresponding $\Delta \mathrm{K}$ values typed in as the scale. 


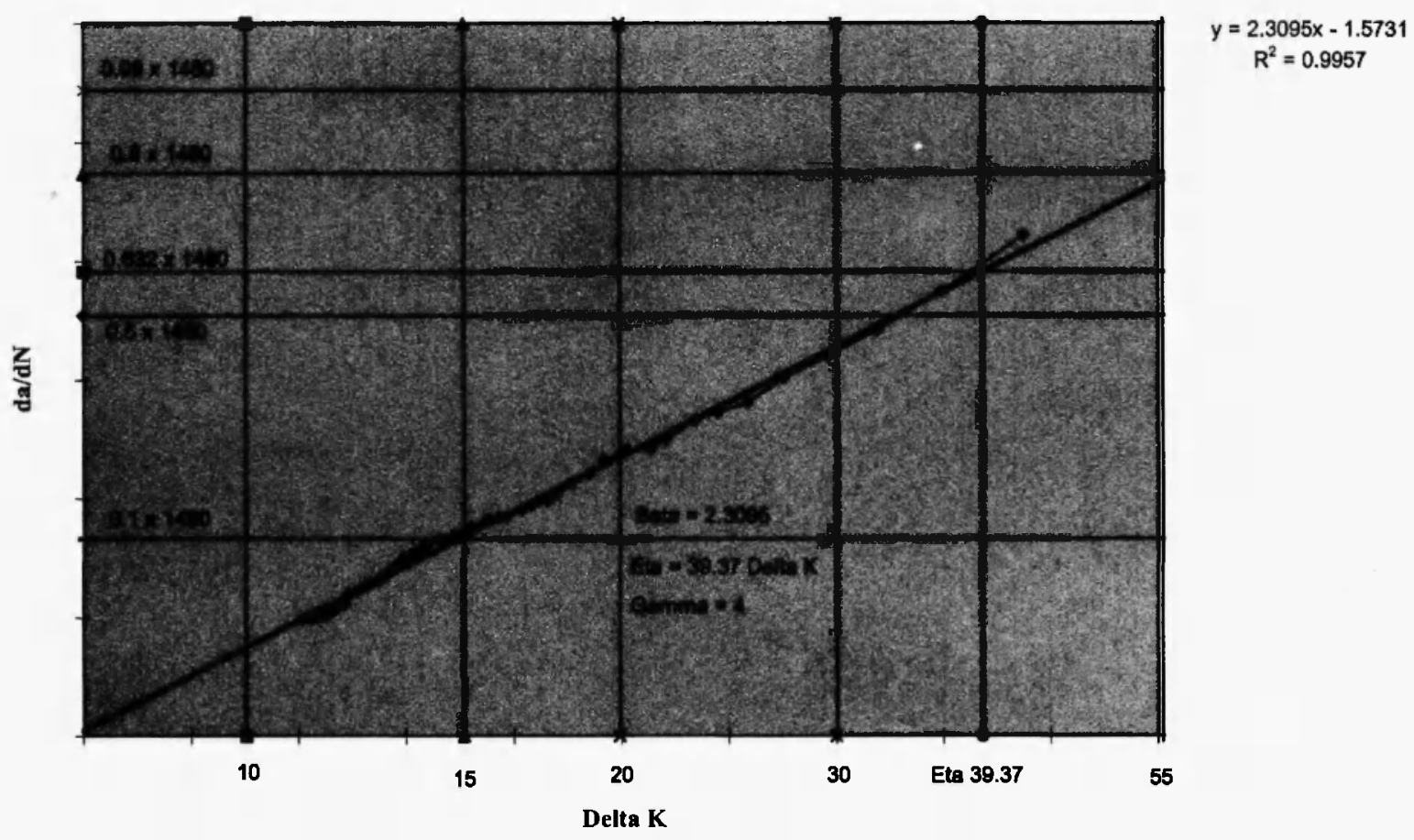

Fig. 2: Weibull plot da/dN vs. Delta $K$

Crack growth rate data is then processed for plotting on the $Y$ axis. The bottom row of columns D \& F contains the final set of data for the test specimen. The highest $\mathrm{da} / \mathrm{dN}$ value (1480) is used as the final member $(100 \%)$ of this pseudo population. This column of data is expressed in column $\mathrm{O}$ as a decimal fraction or percentage of the highest $\mathrm{da} / \mathrm{dN}$ value. This provides a pseudo "failed fraction of a population" value.

The data (except for the final point) is then further processed from right to left through columns $N, M, L$, and $K$ to $J$ which contains the $Y$ axis values. Since column $O$ is the failed fraction column $(F(t))$, the compliment of column $O$ is the surviving fraction or a "Reliability" number $(R)$. Column $N$ is $100 /(100-F(t))$ from the adjacent column $\mathrm{O}$ and is thus labeled $1 / \mathrm{R}$. The $100 \%$ point cannot be processed here because it results in dividing by zero. The next two columns to the left successively provide the natural log of the column to their immediate right. Column $\mathrm{K}$, which is plotted as the "Y" value simply shifts the data scale for presentation as the Weibull Failure Fraction $F(t)$. The $Y$ axis scale construction is shown in Table III. As with the $\mathrm{X}$ axis, the scale values for $\mathrm{f}(\mathrm{da} / \mathrm{dN})$ are suppressed and the Weibull $F(t)$ scale is inserted as data. The scale is then manually labeled.

\subsection{Plotting and Weibull Parameter Determination}

Columns $\mathrm{J}$ and $\mathrm{K}$ are plotted (Figure 2) as an X-Y scatter plot. The Excel "Insert Trend Line" function is 
Table III

Weibull Paper Y Axis Parameters

\begin{tabular}{|c|c|l|}
\hline $\mathrm{F}(\mathrm{t})$ & In In $\{1 /(1-\mathrm{F}(\mathrm{t}))\}$ & $\begin{array}{l}\text { Shifted Column 2 Value for } \\
\text { Weibull } F(\mathrm{t}) \text { plot (Column 2 } \\
\text { minus minimum Column 2 value } \\
(-6.91))\end{array}$ \\
\hline 0.001 & -6.91 & 0.00 \\
\hline 0.01 & -4.60 & 2.31 \\
\hline 0.1 & -2.25 & 4.66 \\
\hline 0.5 & -0.37 & 6.54 \\
\hline 0.9 & 0.83 & 7.74 \\
\hline 0.99 & 1.53 & 8.44 \\
\hline 0.999 & 1.93 & 8.84 \\
\hline
\end{tabular}

then used to obtain a linear trend line and the associated equation. At this point the $\gamma$ value is varied to produce the straightest line of plotted data as indicated by the $R^{2}$ value. The $R^{2}$ value peaked at 0.9957 with a $\gamma$ value of 4 as compared to an initial value $(\gamma=0)$ of 0.9947 . In the resulting equation with $\gamma=4$, the coefficient of $X(2.3095)$ is the value of the Weibull parameter $\beta$. The $\beta$ value with $\gamma=0$ was 2.8

Using the definition of $\eta$ as $F(t)=63.2 \%$, this value is inserted into the last row of the spreadsheet in column $\mathrm{O}$ and processed to the left to column $\mathrm{K}$ just as the actual da/dN data to obtain the $\mathrm{Y}$ axis value of the $63.2 \%$ point. Given this $\mathrm{Y}$ value, the straight line equation provided by the trend line is used in the bottom cell of column $\mathrm{J}$ to solve for the corsesponding $\mathrm{X}$ value. This $\mathrm{X}$ value represents the natural $\log$ of $\eta$. Raising epsilon to this power then provides the $\Delta K$ value of $\eta$ (39.37) displayed in the bottom row of column $D$.

The Weibull PDF and CDF plots produced by the Excel Weibull function using these values are included as Figure 3.

\section{$3.2 \gamma$ or Offset Determination}

The data for specimen CC6 covers both Regions 1 and II. Figure 4 was produced by processing CC6 data with varying values of offset to demonstrate the effect of offset on the shape of the curve. The value of offset providing the straightest lower section of the curve is speculated to be the threshold value of $\Delta \mathrm{K}\left(\Delta \mathrm{K}_{\mathrm{th}}\right)$ for initiation of crack growth. In instances where the data was sufficient to define threshold $\Delta \mathrm{K}$, this would not be of interest. However, in instances where the data has Region I content but not enough to clearly define $\Delta \mathrm{K}_{\mathrm{th}}$, this technique may be of interest. 


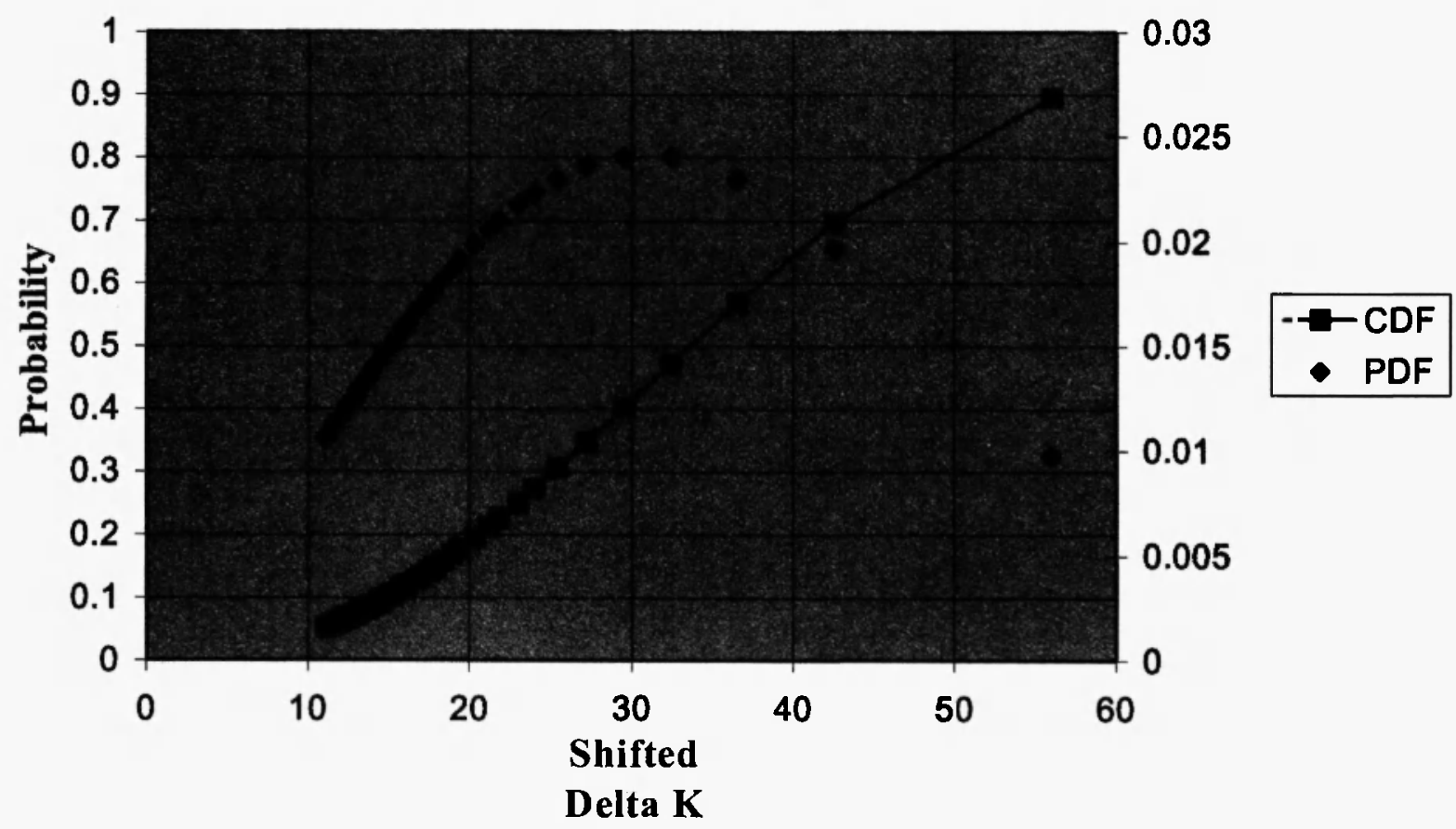

Fig. 3: Weibull da/dN PDF/CDF

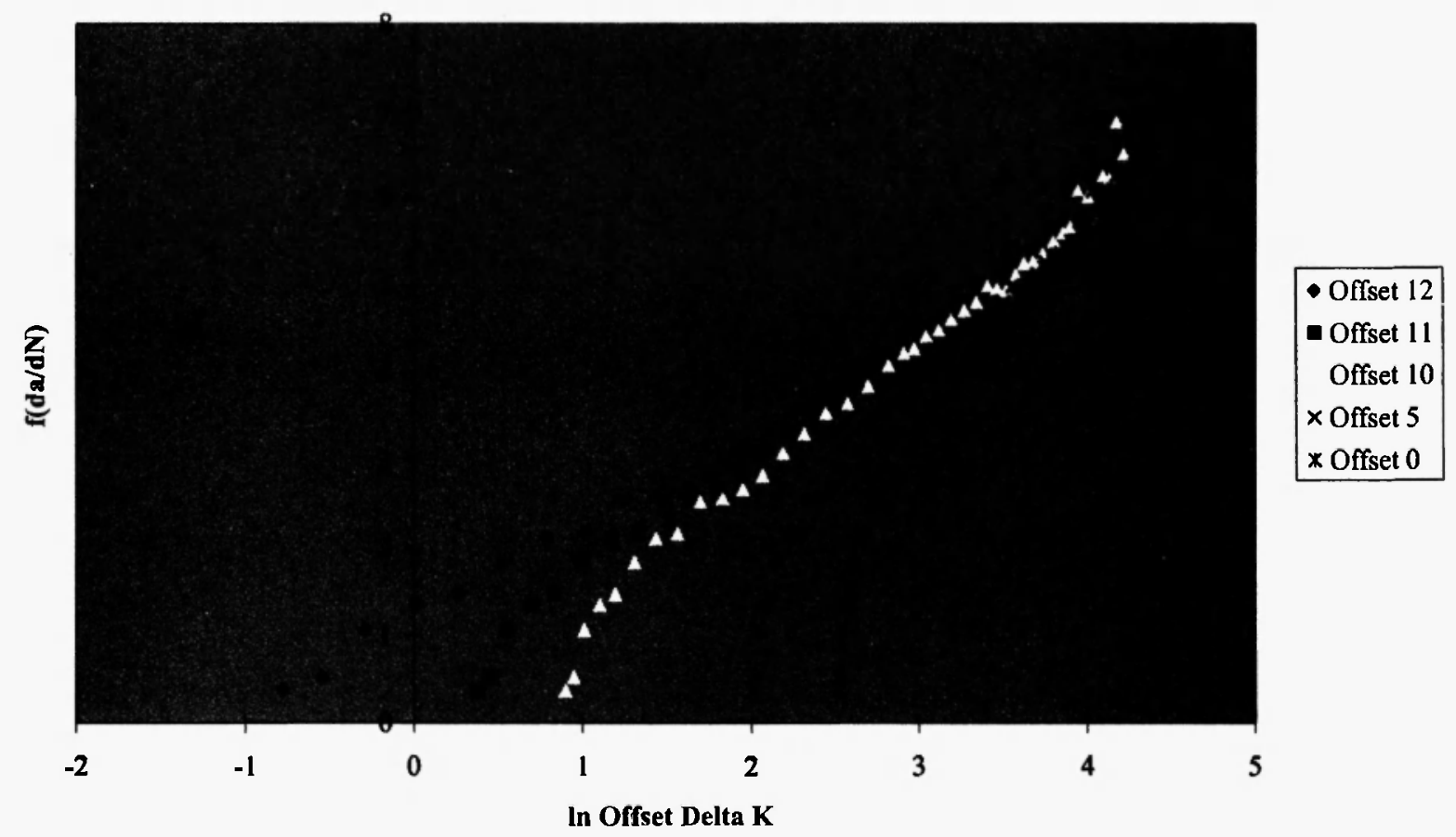

Fig. 4: Offset effect 


\subsection{Composite Results Plot}

Figure 5 is a composite plot of all Weibull plots produced for the 28 sets of usable data. A consistent slope ( $\beta$ parameter) is apparent while a large range of $\Delta K$ for the same $d a / d N$ is evident. This results in a range of the $\eta$ parameter from mid twenties to around 80 . Groupings of trends from closely numbered data sets were noted during plotting. Specimen geometry groupings were also noted with "corner crack" specimens generally lying below and to the right of "compact tensile" specimens. Figures 6 and 7 present the same information as Figure 5 but separated by specimen type.

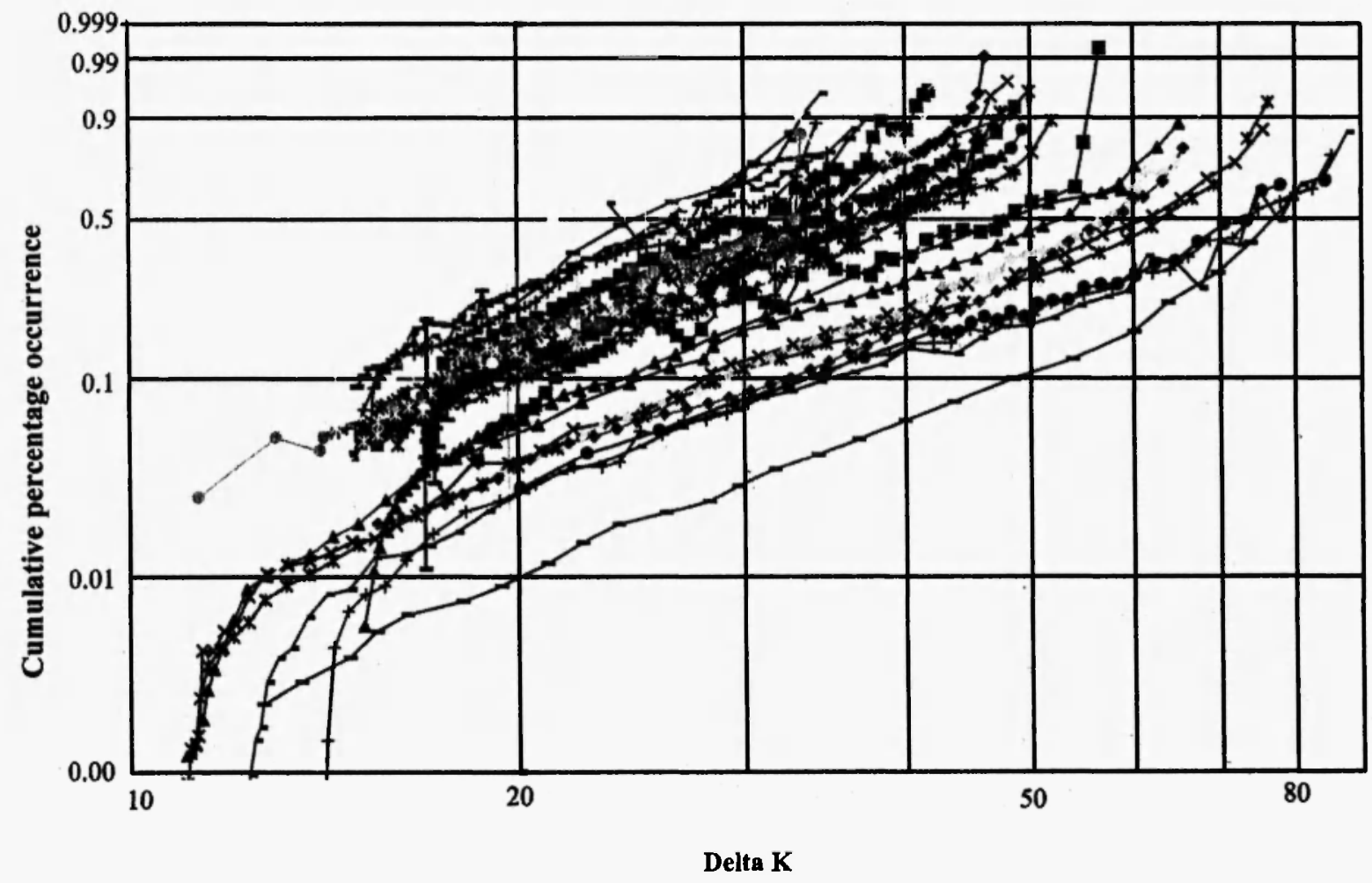

Fig. 5: Weibull composite chart 


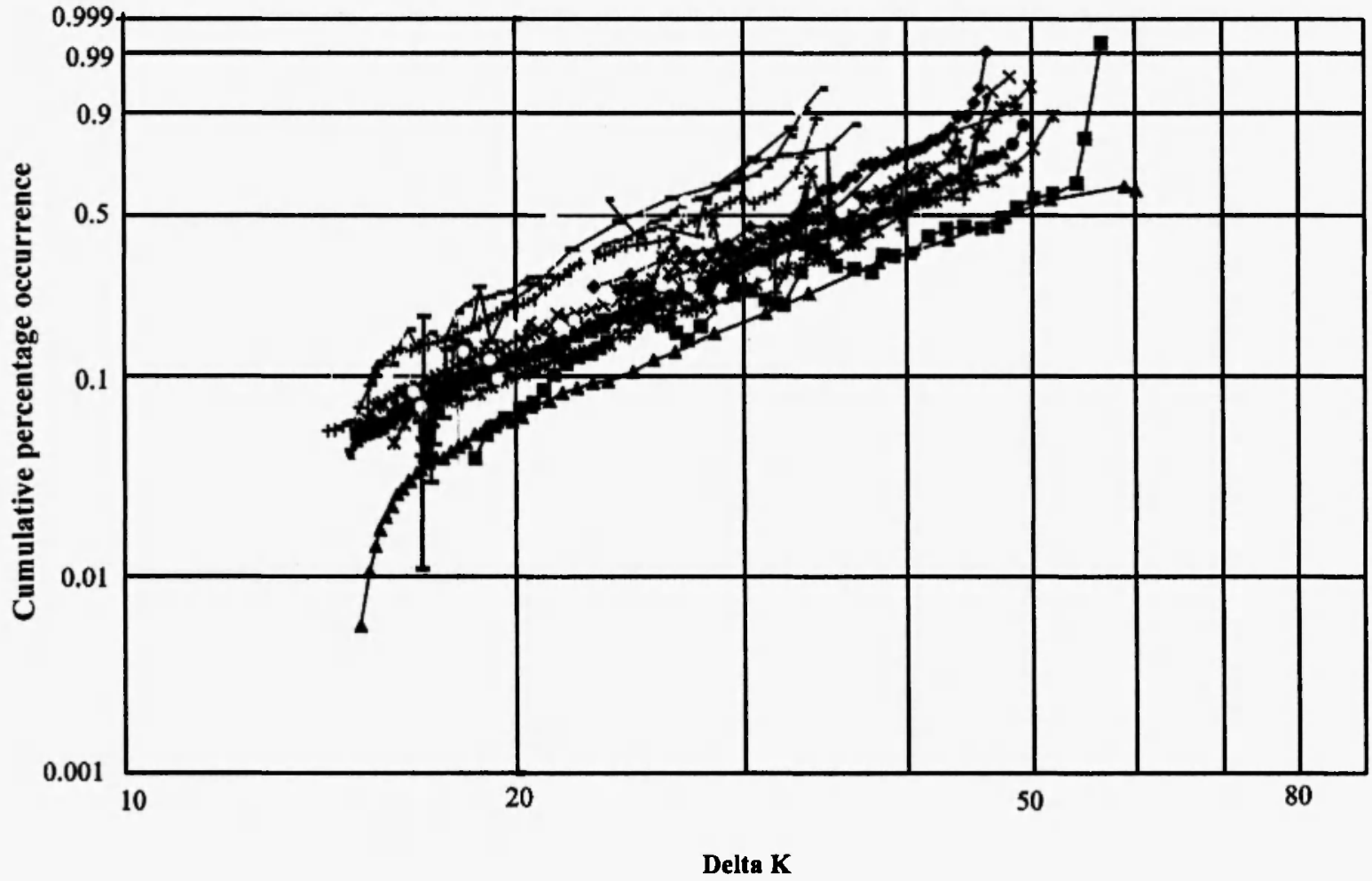

Fig. 6: Compact tensile specimen Weibull plots

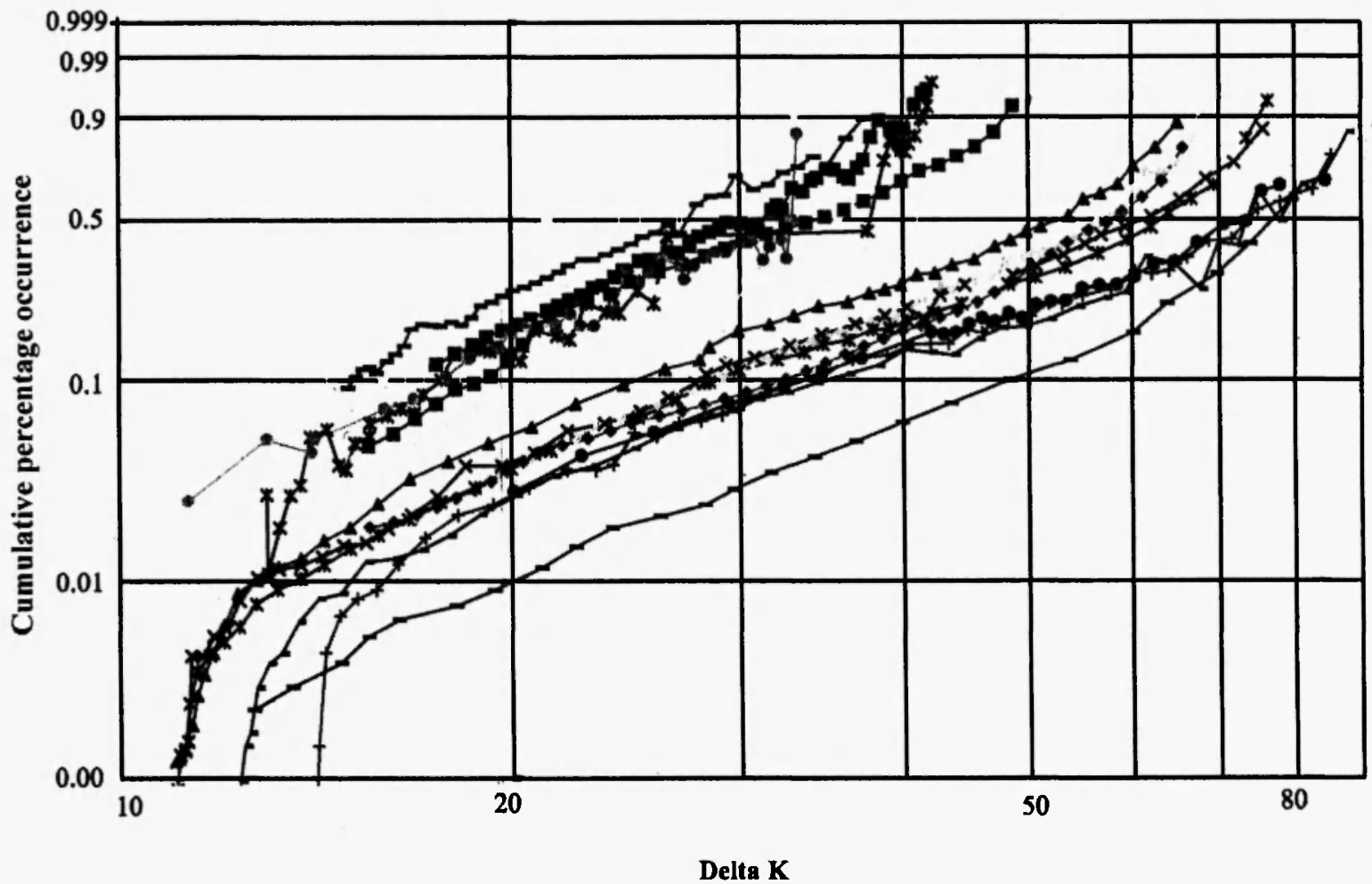

Fig. 7: Corner crack specimen Weibull plots 


\section{CONCLUSIONS:}

1. The methodology was successfully applied to describe the fatigue crack growth rates within Mode 1 stress intensity factor range $(\Delta K)$.

2. The Weibull distribution predicted crack growth rate probability as a function of Mode I $\Delta \mathrm{K}$. In most cases, a straight line fit was observed.

3. From the limited data assessed with the methodology developed in this paper, mixed results were achieved.

4. Additional work is recommended to refine the concepts to develop a more general approach applicable to crack growth rate predictions.

\section{ACKNOWLEDGMENTS:}

Firestone Tube Company provided a stipend to one of the authors (Bobby Day) to develop this methodology. Dr. Scott Jordan, Associate Professor of Mathematics, Arkansas Tech. University provided consultation in development of the procedure.

\section{REFERENCES}

1. A. Weibull, Analysis Handbook AFWAL-TR-83-2079, Abernathy, Breneman, Medlin, \& Reinman, November, 1983.

2. Mechanical Engineering Design, Sixth Edition, J. Shigley \& C. Mishke, McGraw-Hill, 2001.

3. A Weibull, Analysis of Fatigue-Crack Propagation Data from a Nuclear Pressure Vessel Steel, G. C. Salivar and D. W. Hoeppner, Engineering Fracture Mechanics, 12, 181-184.

4. Numerical Modeling of Fatigue and Crack Propagation Test Results by G. E. Bowic and D. W. Hoeppner, International Conference on Computer Simulation for Materials Applications, R.J. Arsenault, J.R. Beeler Jr. and J.A. Simmons (Eds.), 1976; p. 1171. 


\section{APPENDIX I}

\section{Weibull Distribution Failed Fraction Equation}

\section{Conversion to Straight Line Expression}

$$
F(t)=1-e^{((t-\gamma) / \eta)^{\wedge} \beta}
$$

Let $\gamma=0$

$$
F(t)=1-e^{-(t / \eta)^{\wedge} \beta}
$$

Add $\mathrm{e}^{-(\mathrm{t} / \mathrm{n})^{\wedge} \beta}$ to each side, Subtract $\mathrm{F}(\mathrm{t})$ from each side

$$
1-F(t)=e^{-\left((t \eta \eta)^{\wedge} \beta\right.}
$$

Eliminate the negative power of e by taking the reciprocal of the left side

$$
1 /(1-F(t))=e^{(t / \eta)^{\wedge} \beta}
$$

Take natural log of each side

$$
\ln \{1 /(1-F(t))\}=(t / \eta)^{\beta}
$$

Take natural log again

$$
\begin{aligned}
& \ln \ln \{1 /(1-F(t))\}=\beta(\ln t)-\beta \ln \eta \\
& Y=M(X)+B
\end{aligned}
$$

\section{nOverview}

$$
F(t)=1-e^{-(t / n)^{\wedge} \beta}
$$

When $t=\eta$

$$
\begin{aligned}
& F(t)=1-e^{-(1)^{\wedge} \beta} \\
& F(t)=1-0.368 \text { (independent of } \beta) \\
& F(t)=0.632
\end{aligned}
$$


Thus $\eta$ represents the time at which $63.2 \%$ of the population is expected to have failed and is known as the "Characteristic Life." 
